\title{
RESISTÊNCIA ESPECÍFICA DO SOLO DE UM POMAR FRUTÍCOLA RELACIONADA AO MANEJO ENTRELINHAS E INTENSIDADE DE TRÁFEGO \\ LAURA DRAGHI ${ }^{1}$, DANIEL JORAJURÍA ${ }^{2}$, CECILIA CERRISOLA ${ }^{3}$, LUIS MÁRQUEZ DELGADO ${ }^{4}$
}

RESUMO: O tráfego necessário à exploração de pomar frutícola tem características que se denominam "tráfego controlado", já que se realiza sempre sobre os mesmos caminhos do espaço entrelinhas. No alto Valle do Rio Negro, Argentina, a essa situação deve ser agregado, como fator de predisposição das sobrecompactações, o fato de que ocorre com freqüência um excesso de irrigação, que obriga ao tráfego sobre um solo úmido e, portanto, com baixa capacidade de suporte e alta predisposição à compactação. O presente trabalho teve o objetivo de estabelecer a possibilidade de sustentabilidade do sistema produtivo, que se conseguiria com a redução do tráfego resultante das mudanças das aplicações fitossanitárias de calendário fixo, pelas propostas no Projeto Frutícola Integrado (PFI) do INTA (Instituto Nacional de Tecnologia Agropecuária). Em um pomar de macieiras, foi simulado, sobre duas condições de solo, com e sem cobertura vegetal, o tráfego correspondente ao somatório de um ano para cada sistema. Para os tratamentos de tráfego, foi utilizado um conjunto trator-pulverizador padrão. $\mathrm{O}$ parâmetro que se vinculou à compactação, foi a resistência à penetração. $\mathrm{O}$ estado do solo anteriormente à aplicação do tráfego foi tomado como testemunha. $\mathrm{A}$ medição de sobrecompactações sobre o solo nu testemunha foi mais freqüente que sobre o coberto por vegetação. Depois dos tratamentos, a reologia dos solos foi diferente. Concluiu-se que as diminuições do tráfego, como as que foram propostas no PFI, são de significativa importância na busca da sustentabilidade do sistema produtivo.

PALAVRAS-CHAVE: compactação dos solos, tráfego do solo, resistência à penetração.

\section{ORCHARD SOIL REOLOGY RELATED TO COVER CROP MANAGEMENT AND TRAFFIC INTENSITIES}

\begin{abstract}
Orchard production traffic demands have characteristics that is called controlled traffic. Tractors and its machines, always use the same tracks in each of their passage. Not only this negative condition, but the fact that the irrigation used is usually excessive, it leads to a highly potential condition to enhance over-compaction in Alto Valle region in Argentina. This paper is part of a thesis refered to the possibility of introducing changes in the manner of the phytosanitary applications are done. These changes consist in leaving the fixed calendar method to the one proposed by the Integrated Orchard Production program of the INTA. In an apple orchard field, on two soil conditions, with and without grass, traffic corresponding to a whole year management passages of a tractor and it sprayer were simulated for both systems. Penetration resistance was assessed before and after traffic, and related to induced compaction. Over-compaction values were measured more frequently on nongrassed soil if control plot was considered. Edaphic reology was different for each soil condition after traffic was applied. Traffic decreases due to Integrated Orchard Production management and were of significant importance if a sustainable system is the target.
\end{abstract}

KEYWORDS: soil compactness, trafficked soil, penetration resistance.

\footnotetext{
${ }^{1}$ Prof. Adjunto, Departamento de Ingeniería Rural, Facultad Ciencias Agrarias y Forestales, Universidad Nacional de La Plata, Casilla de correos 31, 1900 La Plata, Argentina, ldraghi@agro.unlp.edu.ar

${ }^{2}$ Prof. Titular, Departamento de Ingeniería Rural, Facultad Ciencias Agrarias y Forestales, Universidad Nacional de La Plata, Casilla de correos 31, 1900 La Plata, Argentina, dajo@ceres.agro.unlp.edu.ar.

${ }^{3}$ Profa. Adjunta, Departamento de Suelos y Aguas. Facultad de Ciencias. Agrarias y Forestales. Universidad Nacional de La Plata, Casilla de correos 31, 1900 La Plata, Argentina

${ }^{4}$ Prof. Titular, Departamento de Ingeniería Rural, E.T.S.I.A., Madrid, España, Imarquez@iru.etsia.upm.es

Recebido pelo Conselho Editorial em: 24-5-2002

Aprovado pelo Conselho Editorial em: 5-5-2005
} 


\section{INTRODUCCIÓN}

La producción frutícola del Alto Valle del río Negro y Neuquén, abarca una superficie aproximada de 100000 ha. El clima es continental, templado árido. Los suelos son de origen aluvial, con perfiles de escaso desarrollo pedogenético. La precipitación anual es de $188 \mathrm{~mm}$ por lo cual todos los productores deben regar sus explotaciones. La disponibilidad de agua es elevada y el riego gravitacional se maneja en forma indiscriminada, determinando muchas veces, que el tránsito vehicular se realice en condiciones de alta humedad (SÁNCHEZ, 1999).

La principal plaga que deben combatir los productores es la carpocapsa (Cydia pomonella L.) y su control se basa en la aplicación de insecticidas de síntesis. La forma de control tradicional, a calendario fijo, implica el pasaje repetido de tractores más equipos pulverizadores en un número de hasta 16 veces al año, resultando una intensidad de tráfico que alcanza los $250 \mathrm{Mg} \mathrm{km} \mathrm{ha}^{-1}$ (DRAGHI et al., 1998). La variabilidad que presentan los conjuntos tractor-apero utilizados, es muy baja. Las máquinas pulverizadoras modales tienen un peso de $20 \mathrm{kN}$ soportado en un eje, portan ruedas de escasas dimensiones y se desplazan a velocidades reducidas.

Los repetidos tratamientos fitosanitarios, y las operaciones de cosecha, sobre suelos muchas veces húmedos, sumado al alto peso que pueden portar las máquinas pulverizadoras y sobre ruedas de escaso tamaño, dan origen a condiciones con las cuales es fácil compactar significativamente los suelos.

El mayor enemigo natural que debe enfrentar la producción del Valle es la ocurrencia de heladas tardías, desde la segunda quincena de septiembre hasta finales de octubre. Este problema es el que induce a que el espacio interfilar de los montes esté siempre desnudo o sin vegetación. Combinando el suelo negro y húmedo por la aplicación de láminas de agua, más la colocación de estufas o simplemente el quemado de neumáticos, se intenta controlar los efectos negativos de las heladas. Esa lucha pasiva, obliga al pasaje repetido de gradas de discos para mantener el suelo sin vegetación, limpio.

El momento de la cosecha de la fruta, es otra instancia donde importantes cargas normales deben ser resistidas por el suelo (DI PRINZIO et al., 1998). Los tractores, a través del elevador hidráulico de tres puntos, transportan cajones de grandes dimensiones con la fruta recogida, obligando a la colocación de numerosos lastres delanteros para evitar la pérdida de dirección de los vehículos.

Hay entonces una serie de condiciones que predisponen la ocurrencia de compactaciones indeseadas, las cuales serán acumulativas con el tiempo debido a la inexistencia de roturaciones periódicas del suelo en el área interfilar, una vez que el monte está implantado.

La cuantificación de los niveles de resistencia mecánica inducida por el tráfico agrícola, es un primer aspecto relevante para poder contribuir en la conservación del recurso suelo. Considerando las altas intensidades de tránsito que requiere la aplicación de productos fitosanitarios, el Instituto Nacional de Tecnología Agropecuaria (INTA) del Alto Valle está llevando adelante el primer proyecto en Latinoamérica de Producción Frutícola Integrada. En 1998, la superficie afectada en el proyecto, fueron 1300 ha superando $70 \mathrm{Mg}$ de fruta producida según esta forma. El objetivo inicial es reducir a ocho el número de pasadas anuales de las máquinas pulverizadoras. Si bien fueron razones sanitarias y ecológicas las planteadas inicialmente, la consecuente densificación determinada por los repetidos pasajes del tractor y la pulverizadora, podría ser una problemática a resolver. Se trabajará sobre la hipótesis de que una menor intensidad de tráfico, como consecuencia de lograr un menor número de tratamientos fitosanitarios y utilizar una plataforma para la cosecha, contribuirá a la sustentabilidad del nuevo sistema productivo, desde el momento que disminuirá los daños que el aumento de la impedancia mecánica ocasiona. 
Numerosos trabajos demuestran que la compactación de los suelos de los montes frutales, afectan tanto la disponibilidad de agua y nutrientes para los árboles, como su desarrollo total y la calidad final de la fruta. El parámetro que más usualmente aparece para cuantificar la compactación, es la resistencia a la penetración (RP). NUÑEZ \& VALDEZ (1994), estudiando el efecto de las condiciones del suelo sobre la productividad de un monte de naranjos, encuentran como condición edáfica común a las zonas de baja producción, una RP que se ubicó en $1528 \mathrm{kPa}$ por encima de aquel medido en las zonas de alta producción. ABERCROMBIE (1990) encontró resultados similares en un huerto de aguacates, demostrando correlaciones entre resistencia a la penetración, vigor de las plantas y crecimiento radicular. Los valores máximos de presiones que pueden ejercer las raíces para desarrollarse satisfactoriamente varían entre 0,9 y 1,5 MPa ( BOWEN et al., 1994) aunque los mismos autores resaltan que inferiores valores de resistencia a la penetración pueden limitar significativamente la extensión radicular. THREADGILL (1982) concluye que valores superiores a 1,5 MPa comprometen o dificultan el normal desarrollo radicular y por encima de 2,5 $\mathrm{MPa}$ el crecimiento puede detenerse.

Referido a la conducción del monte frutal, emerge como importante el manejo del monte en el espacio remanente entre las hileras de árboles (interfilar). Este puede incluir prácticas que van desde la eliminación de toda la vegetación herbácea existente, hasta la implantación de diferentes coberturas, pasando por el mantenimiento de las especies vegetales nativas del lugar. Los objetivos buscados al permitir la existencia de coberturas verdes, son varios. Según ELMORE (1989) se intenta contar con un medio que permita la conservación del recurso suelo y del agua y que mejore la capacidad de infiltración. Además se intenta prevenir el encostramiento del suelo y reducir el uso de fertilizantes nitrogenados con la implantación de especies leguminosas. Las coberturas vegetales protegen a los agregados superficiales de su posible disrupción, producto de los impactos del agua de lluvia y/ó de las láminas de agua aplicadas (FOLORUNSO et al., 1992). Cuando los suelos son cultivados por varios y repetidos años con coberturas verdes, necesariamente el aumento en el nivel de materia orgánica mejora la estabilidad estructural y la infiltración, aunque dichos efectos pueden no ser visualizados en los primeros dos años de implantación (MOORE et al., 1989).

Este trabajo se fundamenta en la hipótesis básica de que los valores previos citados por varios autores, de resistencia a la penetración, son efectivamente responsables por mermas o detención del crecimiento de las raíces de los árboles frutales. Las hipótesis de trabajo son: 1.- La reología del suelo es diferente cuando se conduce el espacio interfilar con vegetación herbácea permanente, con respecto a cuando se lo hace a suelo desnudo. 2.- La disminución del tráfico utilizado en el sistema de control fitosanitario a calendario fijo hasta valores emergentes de la propuesta del Proyecto Frutícola Integrado, disminuye los niveles de impedancia mecánica.

\section{MATERIALES Y MÉTODOS}

El ensayo se instaló en un huerto de manzanos de la Estación Experimental Agrícola "Alto Valle", del Instituto Nacional de Tecnología Agropecuaria, en la Provincia de Río Negro, localidad de General Roca. Los árboles fueron implantados en el año 1994 en un marco de 4 x 2 m. El sistema de riego utilizado es con microaspersores, sistema mecanizado de doble propósito: riego y control de heladas. La mitad del espacio interfilar del huerto fue sembrado con festuca (Festuca arundinacea) como cultivo permanente. La otra mitad, no fue sembrada, realizándose un control mecánico de las malezas mediante repetidos pasajes con grada de discos.

\section{Del suelo}

Clasificado como Aquícambids fluventico (Soil Taxonomy, 1994).

Descripción del perfil: 
Horizonte A. (0-22 cm); franco, friable a firme en húmedo; levemente carbonatado; presencia moderada de raicillas; límite claro y abrupto.

Horizonte Bw. (22-35 cm); franco arcillo limoso; prismas medios tendencia bloques angulares, firme en húmedo, barnices finos y discontinuos; levemente carbonatado; presencia de algunas raicillas.

Horizonte C1k. (35-55 cm); franco limoso; bloques angulares y subangulares, medios débiles; friable en húmedo, muy carbonatado, presencia de algunas raicillas.

Horizonte C2. $(55-70 \mathrm{~cm})$; franco arenoso fino; reacción positiva al alfa-alfa dipiridilo.

Profundidad de la napa freática: $132 \mathrm{~cm}$.

\section{Del conjunto tractor-apero}

Tractor Same con tracción delantera asistida FWA (ASAE Standards), de $45 \mathrm{~kW}$ de potencia. Masa total: $2.400 \mathrm{~kg}$. Rodados traseros 12,4-28, de carcasa diagonal, presión de inflado: $152 \mathrm{kPa}$. Rodados delanteros: 8,00-20, presión de inflado: $172 \mathrm{kPa}$

Pulverizadora Pazima, hidroneumática de chorro proyectado. Masa total: $2.700 \mathrm{~kg}$.

Capacidad nominal: 1930 L. Rodados: 7,50-20, presión de inflado: 227 kPa.

\section{De la modelización del tráfico}

Se calcularon previamente las intensidades de tráfico para simular las operaciones de un ciclo anual del cultivo, resultando que el conjunto tractor-pulverizadora debería pasar 27 veces para simular el manejo convencional y 11 veces según la propuesta de producción integrada. Los tratamientos obedecieron a la necesidad de contrastar el manejo tradicional de los huertos, que implica una gran intensidad de tráfico siempre sobre suelo desnudo, con la alternativa impulsada por el INTA para la producción integrada que disminuye el número de pasadas y requiere cobertura verde en el espacio interfilar. Se consideraron como tratamientos, las dos condiciones de suelo (empastado y desnudo), que recibieron dos intensidades de tráfico, la menor $\left(145,33 \mathrm{Mg} \mathrm{km} \mathrm{ha}^{-1}\right)$ que es la que corresponde a la resultante anual toda vez que se utilice el sistema de manejo propuesto en el marco del PFI, y por otro lado la mayor $\left(347,23 \mathrm{Mg} \mathrm{km} \mathrm{ha}^{-1}\right)$ correspondiente al manejo tradicional predominante. Se agregó además la evaluación sobre una parcela sin tráfico que sirvió de testigo. Así resultaron los siguientes subtratamientos:

TABLA 1. Subtratamientos aplicados según intensidades de tráfico y condiciones del suelo.

\begin{tabular}{cccc}
\hline Subtratamiento & Condición Suelo & Número de Pasadas & IT $\left(\mathrm{Mg} \mathrm{km} \mathrm{ha}^{-1}\right)$ \\
\hline 1 & empastado & 0 (testigo) & 0 \\
2 & empastado & 11 & 145,33 \\
3 & empastado & 27 & 347,23 \\
4 & desnudo & 0 (testigo) & 0 \\
5 & desnudo & 11 & 145,33 \\
6 & desnudo & 27 & 347,23 \\
\hline
\end{tabular}

Cada subtratamiento tuvo tres repeticiones totalmente al azar dentro de los dos sectores del huerto.

Para cuantificar la resistencia a la penetración se utilizó un penetrómetro Agridry Rimyk, modelo CP20, con datta logger incorporado y medición de la profundidad por ultrasonido. Se tomaron lecturas cada $25 \mathrm{~mm}$, desde la superficie hasta una profundidad máxima de $575 \mathrm{~mm}$. En cada subtratamiento se realizaron treinta repeticiones.

En cada subtratamiento se determinó el valor de humedad gravimétrica para distintas profundidades del perfil. 


\section{RESULTADOS Y DISCUSIÓN}

\section{Condición mecánica de los suelos testigo}

Para analizar comparativamente los datos de resistencia a la penetración en ambas condiciones de suelo, se constató que no hubieran diferencias significativas de humedad edáfica entre los tratamientos (Tabla 2).

TABLA 2. Contenidos de humedad gravimétrica de las muestras extraídas con cilindro muestreador para distintas profundidades. Media de tres repeticiones en cada subtratamiento.

\begin{tabular}{cccc}
\hline \multirow{2}{*}{ Subtratamiento } & \multicolumn{3}{c}{ Humedad Edáfica $(\%) \mathrm{p} / \mathrm{p}$} \\
\cline { 2 - 4 } & $150 \mathrm{~mm}$ & $300 \mathrm{~mm}$ & $500 \mathrm{~mm}$ \\
\hline 1 & 25 & 25 & 28 \\
2 & 23 & 25 & 26 \\
3 & 26 & 23 & 28 \\
4 & 24 & 24 & 30 \\
5 & 22 & 23 & 28 \\
6 & 22 & 24 & 29 \\
\hline
\end{tabular}

Luego de esta confirmación, se analizó la condición mecánica de los suelos testigos, que refleja el impacto del tráfico acumulado desde la implantación del monte. En el suelo desnudo, los valores testigos de resistencia a la penetración, fueron muy elevados, llegando a superarse la capacidad de lectura del penetrómetro. Del total de datos relevados disponibles, el 37\% superaron $5 \mathrm{MPa}$. Luego de recibir el pasaje del tractor y la pulverizadora, nuevamente superaron la capacidad máxima de medición del aparato el $42 \%$ de los valores, para la menor intensidad de tráfico y el $44 \%$ para aquella equivalente al manejo tradicional de los productores. La profundidad donde mayormente se registraron estos valores, que según la bibliografía antes citada, impedirían el normal crecimiento radicular, se localizó entre los 100 y $125 \mathrm{~mm}$.

En la condición de suelo con cobertura vegetal, esta situación no se presentó nunca, lo que ya evidencia una condición inicial muy diferente entre los dos tratamientos ensayados, lo que demuestra un comportamiento no uniforme (Figura 1).

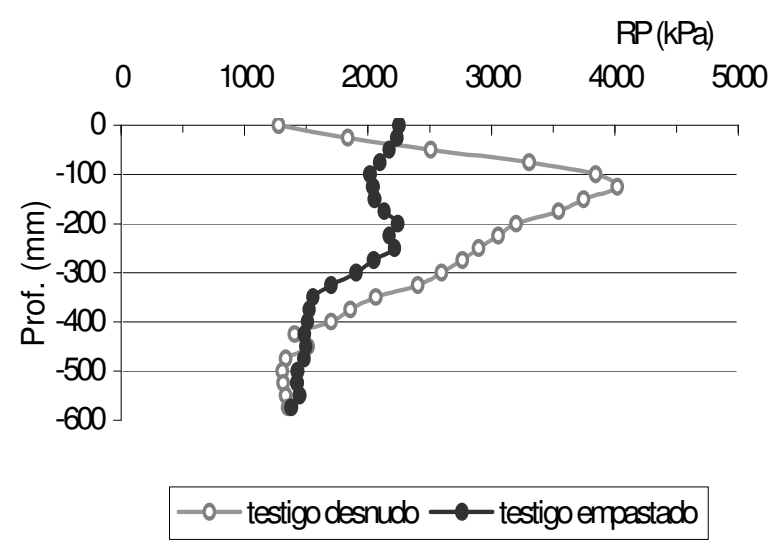

FIGURA 1. Valores de resistencia a la penetración $(\mathrm{kPa})$ registrados en los tratamientos testigos (sin tráfico) para las distintas profundidades (mm).

Sólo en los primeros $25 \mathrm{~mm}$ de profundidad, la resistencia a la penetración del suelo testigo con cobertura vegetal, fue superior a la del terreno desnudo. Esta situación es fácilmente explicable por el pasaje repetido de una grada de casquetes que mantiene el suelo limpio y removido superficialmente. 
A partir de los $50 \mathrm{~mm}$ y hasta los $400 \mathrm{~mm}$ de profundidad inclusive, la resistencia a la penetración del testigo desnudo, fue superior a la del terreno cubierto. La hipótesis explicativa podría ser que la cobertura verde y sus raíces son capaces de brindar una mayor capacidad portante a nivel superficial y el suelo, soportar mejor los sucesivos pasajes.

El suelo testigo sin cobertura vegetal tiene un promedio en todo el perfil estudiado de 2,34 $\mathrm{MPa}$, mientras que el empastado sólo alcanza 1,83 MPa. Si bien esto implica una diferencia cercana al $28 \%$ entre los valores de los suelos testigo, aún se ubica muy por debajo de los 1,5 MPa citados por NUÑEZ \& VALDEZ, (1993), como diferencia de la resistencia a la penetración que marcaba el pasaje a zonas de baja producción.

El valor promedio de RP del suelo testigo desnudo, está un $134 \%$ por encima del límite encontrado por NARRO FARÍAS (1994), para un adecuado crecimiento radicular y dentro del rango citado por THREADGILL (1982), como responsable del impedimento del crecimiento radicular de frutales (2,1 a 2,5 MPa); en el suelo con pasto nunca se alcanzan estos valores. En este mismo sentido, según los datos experimentales aportados por BLANCHER et al. (1978), el suelo desnudo estaría un $17 \%$ por encima del umbral que garantiza el crecimiento (2 $\mathrm{MPa})$, y el suelo con pasto un $9 \%$ por debajo.

Hasta los $400 \mathrm{~mm}$ de profundidad, perfil donde se concentran la mayoría de las raíces, el suelo desnudo tiene un valor promedio de $2,7 \mathrm{MPa}$, condición mecánica que resultaría determinante de la detención del crecimiento radical (THREADGILL, 1982). Para el tratamiento empastado, no se alcanzan valores tan elevados, pero igualmente, al superar 1,5 $\mathrm{MPa}$, las condiciones alcanzadas son limitantes para el óptimo desarrollo de las raíces. Esto demuestra que, aunque la masa de los equipos que transitan normalmente los huertos, apenas sobrepasa los $5 \mathrm{Mg}$, las muy altas intensidades de tráfico recibidas por numerosos pasajes, comprometen capas profundas del perfil, en contraposición a lo encontrado por VORHHEES (1989). Esta situación fue relatada por JORAJURÍA et al. (1997) quienes encontraron que el pasaje repetido de un tractor de escasa masa, puede inducir sobrecompactaciones por debajo del horizonte arable.

\section{Incidencia del tráfico aplicado}

Las dos intensidades de tráfico ensayadas sobre el suelo desnudo, provocaron en superficie los mayores valores absolutos de resistencia a la penetración superando significativamente a todos los demás tratamientos y aumentando el valor inicial del testigo en un $183 \%$ en promedio (Figura 2).

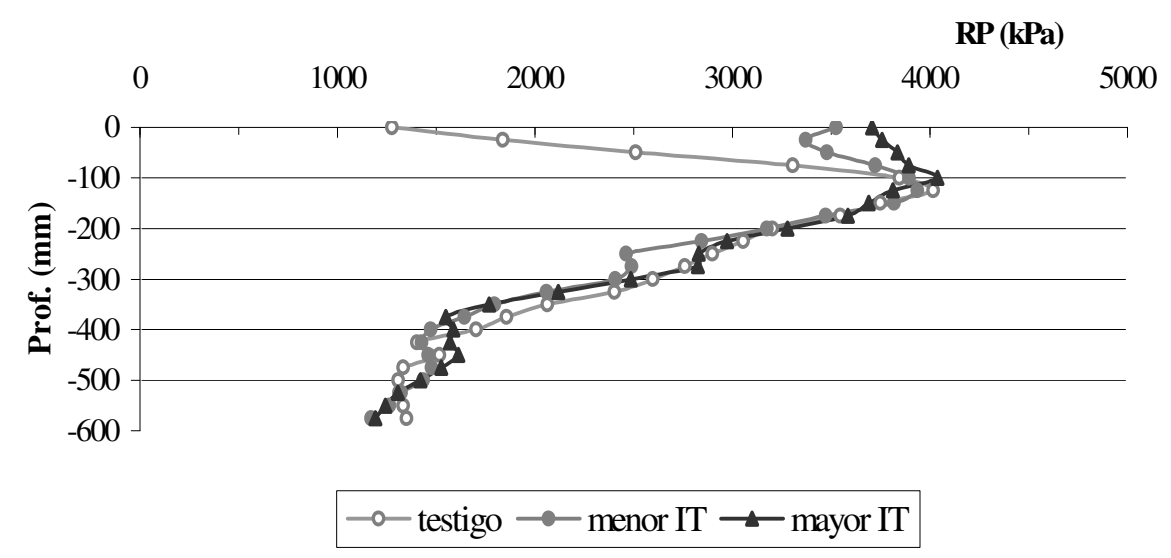

FIGURA 2. Valores de resistencia a la penetración según las diferentes profundidades en los distintos tratamientos sobre el suelo desnudo. 
Es en los $50 \mathrm{~mm}$ donde se observó que el suelo testigo desnudo, incrementó su resistencia a la penetración significativamente, para ambas intensidades de tráfico, alcanzando valores cercanos a 4 $\mathrm{MPa}$, cuando se circuló 27 veces. El mismo comportamiento ocurrió a los $75 \mathrm{~mm}$ de profundidad. Hasta esta profundidad, el suelo se mostró muy susceptible a ambas intensidades de tráfico y los mayores valores siempre correspondieron a la máxima intensidad de tráfico ensayada. Luego, presumiblemente debido a los muy elevados valores iniciales de resistencia a la penetración fue difícil registrar modificaciones. A partir de los 300 mm nunca se modificó la condición inicial.

El tráfico sobre el pasto también aumentó en superficie la resistencia a la penetración inicial, siendo el incremento, en el caso de la menor intensidad de tráfico superior al 40\% (Figura 3). Si bien el tratamiento 3 aumentó el valor del testigo en un 15\%, no evidenció diferencias en el análisis estadístico al $1 \%$ de probabilidad. Este resultado inesperado, podría explicarse si se evalúa el comportamiento de la humedad edáfica volumétrica $(\mathrm{H} v / \mathrm{v} 35,1 \%)$. Presumiblemente, sea éste el factor determinante del menor valor de resistencia a la penetración registrado. Al transitar el suelo con una intensidad de tráfico tan elevada, los agregados podrían haber sufrido una especie de "amasado", con desaparición de gran parte del espacio poroso de aireación, resultando entonces que la proporción del volumen de agua en el volumen total del suelo, haya aumentado. El suelo, que tenía inicialmente un valor de $\mathrm{H}(\mathrm{v} / \mathrm{v})$ de $30 \%$, aumentó hasta el 35\% cuando recibió la máxima intensidad de tráfico ensayada. Esta hipótesis explicativa es coincidente con lo expuesto por JORAJURÍA et al. (1995) quienes encontraron que el tránsito sobre un suelo fino, expulsa rápidamente el aire, pero no el agua, resultando en una mayor humedad volumétrica y menores valores de resistencia a la penetración, aunque mayores valores de densidad aparente. Puede verse cómo entre los $175 \mathrm{~mm}$ y $275 \mathrm{~mm}$ aunque exista pasto, el manejo tradicional del huerto frutal, aumentó significativamente la resistencia a la penetración inicial alcanzando valores aproximados a los 2,5 $\mathrm{MPa}$, mientras que con la menor intensidad de tráfico el valor del testigo no se incrementó y tampoco lo hizo en las demás profundidades analizadas. A partir de los $300 \mathrm{~mm}$, la condición inicial de resistencia a la penetración no fue modificada.

Luego de los $500 \mathrm{~mm}$, ningún tratamiento sobre ninguna de las dos condiciones de suelo presente (desnudo y empastado) alcanzó un valor de resistencia a la penetración superior a 1,5 MPa.

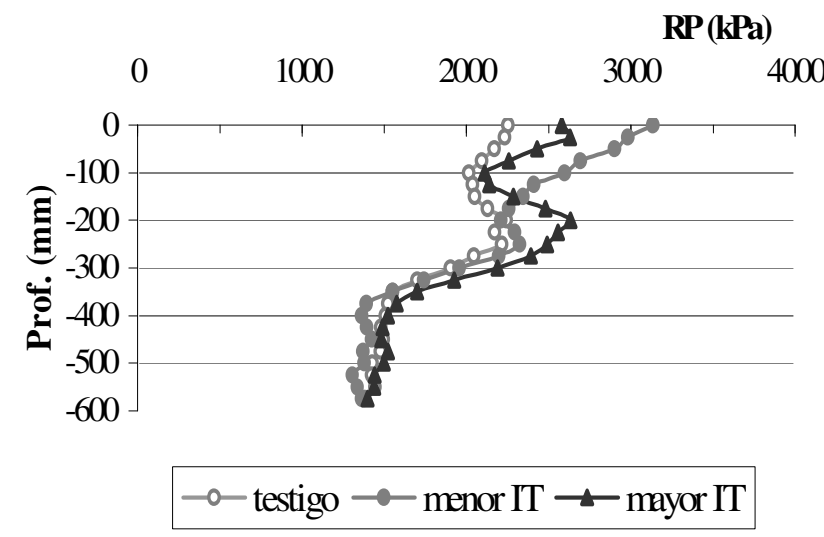

FIGURA 3. Valores de resistencia a la penetración según las diferentes profundidades en los distintos tratamientos sobre el suelo con pasto.

Debido a que el monte ha recibido un nivel alto de tráfico desde que se lo implantó, se realizaron mediciones con el penetrómetro en la línea de plantación de los frutales, en el espacio entre árboles consecutivos. Estos valores reflejan la situación mecánica del suelo que sólo ha recibido consolidación pero no ha sido afectado por el tráfico repetido. En primer lugar, el valor promedio de RP de todo el perfil estudiado alcanzó 1,5 MPa. El suelo desnudo presenta valores de resistencia mecánica igual a la 
condición de suelo consolidado a los $350 \mathrm{~mm}$, mientras que en el terreno empastado ya se alcanzó a los $250 \mathrm{~mm}$ (Figura 4). Por lo tanto hay un mayor volumen de suelo que ha modificado su resistencia mecánica si el tránsito se ha realizado sobre suelo desnudo. Puede entonces inferirse que el pasto ha soportado mejor el tránsito a lo largo de los años.

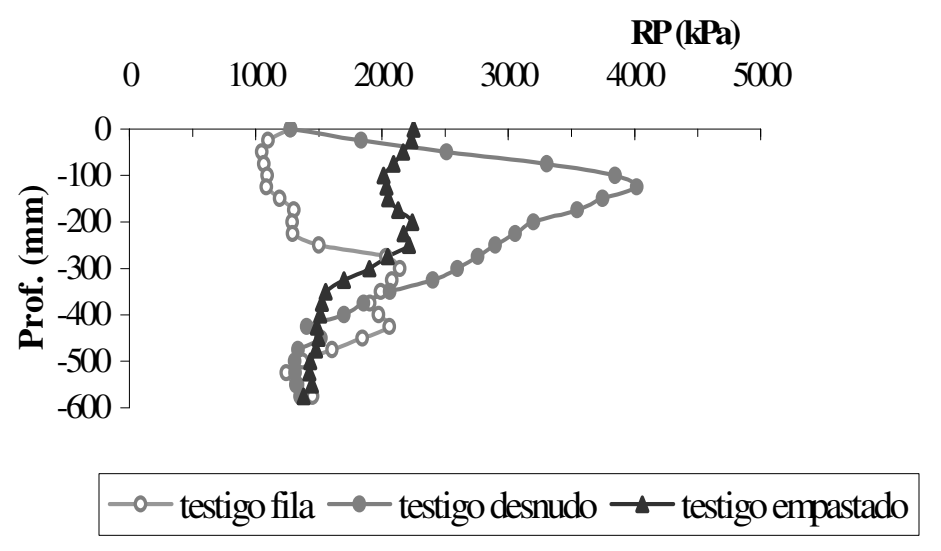

FIGURA 4. Comparación de los valores de resistencia a la penetración del suelo consolidado con respecto al que ha recibido tráfico.

En el suelo desnudo, los incrementos productos del tráfico repetido, son siempre muy superiores a la situación del suelo empastado superando el $100 \%$ hasta los $225 \mathrm{~mm}$ de profundidad, estrato donde el suelo empastado ya no detecta diferencias estadísticas significativas. Esto, sumado al hecho que el testigo con cobertura verde, en el momento de la toma de datos, presentó una resistencia a la penetración siempre inferior al suelo desnudo ya a partir de los $50 \mathrm{~mm}$, permitiría inferir, una vez más, el mejor comportamiento del pasto a la hora de tener que soportar altas intensidades de tráfico.

Por último, se revisaron las profundidades adonde se registraron valores de resistencia a la penetración superiores a los 2,5 $\mathrm{MPa}$ ya que este valor es considerado como freno ó causante de la detención del crecimiento radicular. El suelo empastado, en su situación testigo, nunca mostró valores de resistencia a la penetración superiores a los 2,5 MPa. La menor intensidad de tráfico ensayada alcanzó valores superiores a los 2,5 $\mathrm{MPa}$ en el intervalo de 0 a $100 \mathrm{~mm}$ de profundidad (el valor promedio fue de 2,8 $\mathrm{MPa}$ ). Cuando la intensidad de tráfico aumentó hasta representar la del manejo tradicional de los huertos, aparecieron valores superiores a los 2,5 $\mathrm{MPa}$ hasta una profundidad mayor, alcanzando los $225 \mathrm{~mm}$, por lo tanto la profundidad afectada se duplicó. El valor promedio en ese intervalo de profundidad fue de 2,6 MPa.

En el suelo desnudo testigo ya aparecieron valores superiores a los 2,5 $\mathrm{MPa}$ entre los 50 y 300 mm. Cuando se los traficó 11 veces, se superó esa resistencia a la penetración en el intervalo de 0 a $225 \mathrm{~mm}$, es decir que se involucraron capas menos profundas. Para la mayor intensidad de tráfico, desde 0 hasta los $275 \mathrm{~mm}$ aparecieron valores de resistencia a la penetración superiores a los 2,5 MPa. Debe remarcarse que ambas intensidades de tráfico, superaron los 3,5 $\mathrm{MPa}$ en esos intervalos de profundidad.

El suelo desnudo mostró valores superiores a los 2,5 $\mathrm{MPa}$ hasta profundidades siempre mayores que el suelo empastado: $100 \mathrm{~mm}$ contra $225 \mathrm{~mm}$ para la menor intensidad de tráfico y $225 \mathrm{~mm}$ versus $275 \mathrm{~mm}$ para la mayor intensidad de tráfico ensayada.

Estos resultados avalan la primera hipótesis, y permiten inferir una reología diferente del suelo conducido con pastura permanente con respecto al desnudo. 
Quedó establecido entonces que las mayores intensidades de tráfico, tanto en el suelo desnudo como empastado, siempre alcanzaron valores de resistencia a la penetración mayores a los 2,5 $\mathrm{MPa}$ hasta profundidades mayores que la menor intensidad de tráfico. En el caso del suelo desnudo esa profundidad alcanzó los $275 \mathrm{~mm}$ y en el caso del suelo empastado los $225 \mathrm{~mm}$. Se coincide entonces con SOMMER \& ALTEMÜLLER (1982) citado por ALAKUKKU (1997), en cuanto a que la mayor intensidad de tráfico ocasiona problemas de compactación a mayores profundidades. Se considera entonces que hay avales como para considerar que una reducción de la intensidad de tráfico como la planteada aquí, redundará en una elevación de la localización del horizonte con una resistencia mecánica equivalente a aquella citada como causante de la detención del crecimiento radicular. Esto significaría, en términos aplicables al manejo del cultivo, que la posible solución a través de un tratamiento mecánico, por lo menos se facilita, y muchas veces será la diferencia entre hacerlo factible o no. En la medida que esa capa se ubique a mayor profundidad, más complicado será plantear un tratamiento de descompactación forzada y menos activos serán los agentes descompactadores naturales. A su vez si esa menor intensidad de tráfico se realiza sobre un suelo con cobertura vegetal, la capa más compactada se ubicará a menor profundidad aún y por ende más factible y económico será su tratamiento.

\section{CONCLUSIONES}

Intensidades de tráfico equivalentes al manejo tradicional del huerto frutal, sobre un suelo desnudo, inducen valores de resistencia a la penetración limitantes para el normal desarrollo radicular.

La presencia de cobertura vegetal interfilar disminuye la susceptibilidad del suelo al incremento de la resistencia mecánica.

La reducción de la intensidad de tráfico planteada, sobre suelo desnudo, no evita llegar a niveles de impedancia mecánica limitantes para una producción sustentable.

La presencia de cobertura vegetal en las sendas traficadas tiene más relevancia en la resistencia a la penetración alcanzada, que la propia intensidad de tráfico requerida.

\section{REFERENCIAS}

ABERCROMBIE, R.A. Root distribution of avocado trees on a sandy loam soil as affected by soil compaction. Acta Horticulturae, The Hague, v.275, p.505-12, 1990.

ALAKUKKU, L. Long-term soil compaction due to High Axle Load Traffic. 1997. 98 f. Thesis (Ph.D.) - Institute of Crop and Soil Science, Agricultural Research Centre of Finland, Jokioinen, 1997.

AMERICAN SOCIETY OF AGRICULTURAL ENGINEERING. Standards. St. Joseph, 1992. 781 p.

BLANCHER, R.W; EDMONDS, C.R.; BRADFORD, J.M. Root growth in cores formed from fragipan and B2 horizons of Hobson soil. Soil Science Society of America Journal, Madison, v.42, n.2, p.437-40, 1978.

BOWEN H.D.; GARNER, T.H.; VAUGHN, D.H. Advances in soil-plant dynamics. In: Advances in soil dynamics. St. Joseph: ASAE, 1994. p.255-80.

DI PRINZIO, A.; JORAJURÍA, D.; BEHMER, S.; AYALA, C.; ARAGÓN, A. Efecto del tráfico de la maquinaria en la distribución de la compactación del monte frutal. In: CONGRESO BRASILEIRO DE ENGENHARIA AGRÍCOLA, 27., 1998, Poços de Caldas. Anais... Lavras: Sociedade Brasileira de Engenharia Agrícola, 1998. (Paper MAA 0-35)

DRAGHI, L.; JORAJURÍA, D.; BOTTA, G.; BALBUENA, R.; ARAGÓN, A.; DI PRINZIO, A. Compactación del suelo en el monte frutal inducida por el tráfico vehicular. In: Ingeniería Rural y 
Mecanización Agraria en el ámbito Latinoamericano. La Plata: Associación Latinoamericana y del Caribe de Ingeniería Agrícola, 1998. p.28-35.

ELMORE, C. Vegetation management systems in almond orchards. California Agricultural, Berkeley, v.43, n.4, p.16-20, 1989

FOLORUNSO, O.; ROLSTON, D.; PRICHARD, T.; LOUIE, D. Cover crops lower soil surface strength, may improve soil permeability. California Agricultural, Berkeley, v.46, n.6, p.26-7, 1992.

JORAJURÍA, D.; DRAGHI, L.; ARAGÓN, A. Compactación del suelo bajo tráfico repetido. Investigación Agraria, Producción y Protección Vegetales, Madrid, v.10, n.3, p.473-83, 1995.

JORAJURÍA, D.; DRAGHI, L.M.. The distribution of soil compaction with depth and the response of a perennial forage crop. Journal of Agricultural Engineering Research, London, v.66, p.261-5, 1997.

MOORE, D.; SINGER, M.; OLSON, W. Improving orchard soil structure and water penetration. California Agricultural, Berkeley, v.43, n.5, p.7- 9, 1989.

NARRO FARÍAS, E. Física de suelos con enfoque agrícola. Buenos Aires: Editorial Trillas, 1994. $181 \mathrm{p}$.

NUÑEZ MORENO, J.H.; VALDEZ GASCÓN, B. Effect of soil condition on orange trees. Communications in Soil Science and Plant Analysis, New York, v.25, n.9-10, p.1747-53, 1994.

SÁNCHEZ, E. Nutrición mineral de frutales de pepita y carozo. General Roca: INTA Estación Experimental Alto Valle de Rio Negro, 1999. 125 p.

THREADGILL, E.D. Residual tillage effects as determined by cone index. Transactions of the ASAE, St. Joseph, v.25, n.4, p.859-963, 867, 1982.

VORHEES, W.B. Root activity related to shallow and deep compaction. In: LARSON, W.; BLAKE, G.; ALLMARAS, R.; VOORHEES, W.; GUPTA, S. Mechanics and related processes in structured agricultural soils. The Netherlands: NATO Applied Science, 1989. p.173-86. 